\title{
COMPARATIVE IN- VITRO EVALUATION OF VANCOMYCIN MINIMUM INHIBITORY CONCENTRATIONBY AGAR DILUTION AND E-STRIP IN METHICILLIN RESISTANT STAPHYLOCOCCUS AUREUS
}

\author{
Sheroze Ilyas, Tehmina Munir, Rabia Sadaf, Mehreen Gilani \\ Army Medical College/National University of Medical Sciences (NUMS) Rawalpindi Pakistan
}

\begin{abstract}
Objective: To compare the in-vitro efficacy by determining Minimum Inhibitory Concentration of Vancomycin using the reference Agar Dilution to the E-Strip in Methicillin Resistant Staphylococcus aureus isolates.

Study Design: Validation study.

Place and Duration of Study: The department of Microbiology Army Medical College/National University of Medical Sciences in collaboration with Pak Emirates Military Hospital Rawalpindi, from Dec 2016 to Dec 2017.

Methodology: Non-duplicate 84 isolates of Methicillin resistant Staphylococcus aureus from various clinical specimens were included in the study. All these isolates were screened for susceptibility to glycopeptide by E-strips method (Bio mérieux) as well as Agar Dilution method, using vancomycin concentrations of 0.25, 0.50, 1.00, 2.00, 4.00 and $8.00 \mu \mathrm{gm} / \mathrm{ml}$ respectively in two fold serial dilutions.

Results: There was an overall agreement on 83 samples by both the methods i.e. 83 were Vancomycin Sensitive Staphylococcus aureus by both methods while one isolate with intermediate resistance to Vancomycin was only detected by Agar Dilution. The sensitivity of the E-strips compared to Agar Dilution was found to be 100\%. The positive predictive value was $98.8 \%$ with a diagnostic accuracy of $98.8 \%$. Specificity and negative predictive value could not be ascertained for E-strips because of the limitation of the method to detect the Vancomycin Intermediate Staphylococcus aureus isolates.

Conclusion: E-strip can be a convenient alternative to the gold standard Agar Dilution but its inability to identify VISA challenges its reliability in determining the Vancomycin resistance in MRSA isolates.
\end{abstract}

Keywords: Agar dilution, E-strip, Minimum inhibitory concentration, Methicillin resistant staphylococcus aureus, Vancomycin intermediate staphylococcus aureus, Vancomycin resistant staphylococcus aureus.

This is an Open Access article distributed under the terms of the Creative Commons Attribution License (http://creativecommons.org/licenses/by/4.0), which permits unrestricted use, distribution, and reproduction in any medium, provided the original work is properly cited.

\section{INTRODUCTION}

Methicillin resistant Staphylococcus aureus is one of the leading causes of death not only in hospital settings but also in the community ${ }^{1}$. This multi-resistant organism confers resistance to all beta-lactam drugs leaving behind limited treatment options for the physician². The drug of choice for such resilient and life-threatening infections is the glycopeptide, Vancomycin ${ }^{3}$. The sensitivity of Vancomycin is determined by its MIC (Minimum Inhibitory Concentration), which can either be calculated by the gold standard Agar Dilution or Broth Micro-Dilution, according to CLSI (Clinical Laboratory and Standards Institute) criterion or by various automated or commercially available

Correspondence: Dr Sheroze Ilyas, House No. 65, Street No. 02, DHA Phase 1, Islamabad Pakistan

Received: 26 Nov 2019; revised received: 12 Feb 2020; accepted: 14 Feb 2020 methods like E-strips 4 . The dis-recommendation of disk diffusion method by CLSI, led to the use of E-strips for MIC calculation of vancomycin in various laboratories around the world, as the gold standard was tedious, laborious and time consuming. The E-strip method has an advantage over other MIC calculating tools, in being easy to interpret and easy to set up as a disk diffusion test $^{5}$. The MIC of Vancomycin should be $\leq 2 \mu \mathrm{g} /$ $\mathrm{ml}$ for susceptible strains, $4-8 \mu \mathrm{g} / \mathrm{ml}$ for intermediate strains and $\geq 16 \mu \mathrm{g} / \mathrm{ml}$ for resistant strains according to the CLSI interpretive criteria (CLSI document M100-S25).

The treatment failure of patients with MRSA infections by Vancomycin is not un-common for organisms having slightly higher MICs (more than $1 \mu \mathrm{g} / \mathrm{ml}$ but $\leq 2 \mu \mathrm{g} / \mathrm{ml}$ ), a phenomena known as MIC creep 6 . So the type of method used for 
calculating MIC remains the critical part of the equation7. Recently there have been reports of emergence of Vancomycin Intermediate Staphylococcus aureus (VISA) and Vancomycin Resistant Staphylococcus aureus (VRSA) around the world8, further emphasizing the need for an accurate MIC calculation 9,10 . It also needs to be evaluated whether the two methods accurately detect VISA /VRSA.

This study compares the E-strip with the reference Agar Dilution for determining vancomycin susceptibility in MRSA isolates. Many studies around the world have evaluated the comparative efficacy of the two methods using E-strip and Agar Dilution but limited work has been done in Pakistan. A study was done in Army Medical College $^{11}$ using only E-strips method to calculate VISA and VRSA rates, however, the results obtained were not compared to any of the reference methods recommended by CLSI. The comparison of the two method in our study, will also enable us to implement the best option for calculating the MIC of vancomycin in our present setup and also at the same time gives us an insight whether E-strip can be a reliable method for detecting VISA/VRSA.

\section{METHODOLOGY}

This is a validation study and was approved by review board of Army Medical College and was carried out at the department of Microbiology, Army Medical College, Rawalpindi, from Dec 2016 to Dec 2017, affiliated with the Pak Emirates Military Hospital Rawalpindi. Sample size was calculated using the WHO calculator based on MRSA's pre-valence. Eighty-four isolates of MRSA obtained from various clinical specimens including pus, sputum, blood, urine, nasobronchial lavage and tips etc, were included in the study. Non-probability convenience sampling technique was used. Quality control strain of Staphylococcus aureus ATCC 29213 was evaluated concurrently with every set of tests.

All MRSA isolates were screened for reduced glycopeptide susceptibility by Agar Dilution method. Bacterial suspensions were prepared from overnight cultures of MRSA on blood Agar and their turbidity was adjusted to 0.5 McFarland standard solution. These suspensions were then spot-inoculated on to Mueller Hinton Agar, containing serial dilutions of Vancomycin, along with the controls. Plates were incubated at $35 \pm 2{ }^{\circ} \mathrm{C}$ for 24 hours. The lowest concentration of Vancomycin that inhibited the bacterial growth after overnight incubation was considered as its MIC.

Susceptibility of MRSA to vancomycin by E-strip method was performed according to the manufacturer's instructions (Bio Merieux France). The bacterial suspension of 0.5 McFarland Standard Inoculum in sterile water was lawned onto Mueller Hinton agar plate. The vancomycin Estrip was placed in the center of the plate and incubated at $35 \pm 2^{\circ} \mathrm{C}$ for 24 hours. Isolates were categorized as susceptible, intermediate or resistant according to the breakpoints published by the CLSI. Data was analyzed using SPSS (Statistical Package for Social Sciences) version 22. Frequency and percentage were documented for qualitative variables like sensitivity, specificity, negative predictive values, positive predictive values and diagnostic accuracy.

\section{RESULTS}

A total of 84 samples $52.6 \%$ were received from OPD and $44 \%$ from Ward, ensuring uniform distribution. Two samples of the MRSA were also received from the ITC. Majority of MRSA were obtained from pus specimens 44 (52.4\%), double lumen tip $14(16.7 \%)$ and pus swabs $10(11.9 \%)$ respectively. The rest of the 16 samples were received from various specimens like ear swabs, urine or high vaginal swab etc. The isolates also had almost uniform gender wise distribution 44 $(52.4 \%)$ male and $40(47.6 \%)$ female. Maximum sampleswere received fromages 55-65 years 31 , (27.3\%) followed by ages < 2 years including infants $12(10.7 \%)$.

The frequency of isolates by Agar Dilution was 12, 71 and 1 for MICs 1.00, 2.00, $4.00 \mu \mathrm{g} / \mathrm{ml}$ respectively, with maximum percentage of isolates $(84.5 \%)$ having MIC of $2 \mu \mathrm{g} / \mathrm{ml}$. Eighty three isolates were VSSA, one isolate was found to be 
a VISA with an MIC of $4 \mu \mathrm{g} / \mathrm{ml}$ while no VRSA was detected by Agar Dilution.There were 7, 24, 21 and 32 number of isolates having MIC of $0.38,0.50,0.75,1.00 \mu \mathrm{g} / \mathrm{ml}$ respectively by E-strip, with maximum percentage (38.1\%) of isolates having an MIC of $1.00 \mu \mathrm{g} / \mathrm{ml}$. Thus, all the isolates were found to be sensitive by E-strip (VSSA) i.e. having an MIC of $\leq 2 \mu \mathrm{g} / \mathrm{ml}$. No VISA or VRSA was picked by the E-strip method.

The comparison of MICs calculated using Estrips and Agar Dilution with 84 MRSA isolates is displayed in table-I. There was an overall agreement on 83 samples by both the methods i.e. 83 were VSSA by both methods while one isolate with intermediate resistance to Vancomycin was only picked by Agar Dilution. Study variables were reported in the form of frequencies and percentages.

\section{DISCUSSION}

The E-strip method is very sensitive technique witha very high diagnostic accuracy. However, the inability to diagnose VISA isolates limits the negative predictive values and specificity of the method, leaving behind the gold standard as the only reliable method for detection of less susceptible strains. High accuracy of E-strip method in terms of its sensitivity has been verified in various other studies such as done by Hsu et al ${ }^{13}$ and Chaudhri et al ${ }^{14}$.

The correlation between Vancomycin treatment failure in MRSA bacteremia and slightly increasing MIC values within the normal range, was first observed by Sakoulas ${ }^{15}$. This phenomena of MIC creep was further elaborated by Wang16, in which 123 isolates in a hospital in Taiwan were studied. About $21.1 \%$ samples showed

Table-I: Vancomycin minimum inhibitory concentration results from E-Test and Agar Dilutions.

\begin{tabular}{|c|c|c|c|c|c|c|c|c|c|}
\hline \multirow[t]{2}{*}{$\begin{array}{l}\text { Test } \\
\text { Method }\end{array}$} & \multirow{2}{*}{$\begin{array}{l}\text { Minimum } \\
\text { Inhibitory } \\
\text { Concentration } \\
(\mu \mathrm{g} / \mathrm{ml})\end{array}$} & \multicolumn{7}{|c|}{$\begin{array}{l}\text { No and Percentage of Methicillin Resistant } \\
\text { Staphylococcus aureus Isolates with Minimum } \\
\text { Inhibitory Concentration from } 0.38 \text { to } 8\end{array}$} & \multirow{2}{*}{$\begin{array}{l}\text { Vancomycin } \\
\text { Intermediate } \\
\text { Staphylococcus } \\
\text { aureus }\end{array}$} \\
\hline & & 0.38 & 0.5 & 0.75 & 1 & 2 & 4 & 8 & \\
\hline \multirow{2}{*}{ E-Strip } & Frequency $(n=84)$ & 7 & 24 & 21 & 32 & - & - & - & - \\
\hline & Percentage (\%) & 8.3 & 28.6 & 25 & 38.1 & - & - & - & - \\
\hline \multirow{2}{*}{$\begin{array}{l}\text { Agar } \\
\text { Dilution }\end{array}$} & Frequency $(\mathrm{n}=84)$ & - & - & - & 12 & 71 & 1 & - & 1 \\
\hline & Percentage (\%) & - & - & - & 14.3 & 84.5 & 1.2 & - & 1.2 \\
\hline
\end{tabular}

Using the standard formulae, the sensitivity of the E-strip compared to Agar Dilution was found to be $100 \%$. The positive predictive value was calculated as $98.8 \%$ and diagnostic accuracy as $98.8 \%$. Specificity and negative predictive value could not be defined for E-strips because of the limitation of the method to detect VISA isolates. The same calculated values are reflected in table-II.

Table-II: Validation study parameters.

\begin{tabular}{|c|c|c|c|}
\hline & \multicolumn{2}{|c|}{$\begin{array}{l}\text { Agar Dilution (Gold } \\
\text { Standard) }\end{array}$} \\
\hline & & $\begin{array}{l}\text { Vancomycin } \\
\text { Sensitive }\end{array}$ & $\begin{array}{l}\text { Vancomycin } \\
\text { Intermediate }\end{array}$ \\
\hline \multirow{2}{*}{$\begin{array}{l}\text { E- } \\
\text { Strip }\end{array}$} & $\begin{array}{l}\text { Vancomycin } \\
\text { Sensitive }\end{array}$ & 83 & 1 \\
\hline & $\begin{array}{l}\text { Vancomycin } \\
\text { Intermediate }\end{array}$ & - & - \\
\hline
\end{tabular}

an MIC of $2 \mu \mathrm{g} / \mathrm{ml}$ while the rest were lower than $2 \mu \mathrm{g} / \mathrm{ml}$. The mortality rate in patients with MRSA bacteremia having an MIC of $2 \mu \mathrm{g} / \mathrm{ml}$ was higher as compared to the ones with lower MICs. This further emphasizes the need for precise Vancomycin MIC calculation using the standard method in order to prevent treatment failure, leading to lowering of the mortality rate. Methods like E-strips might not accurately detect the MICs and leave VISA / VRSA undetected.

A similar study in the region ${ }^{5}$ comparing the two methods, showed higher MICsdetected by E-strips as compared to Agar Dilution in which only three isolates out of 53 showed similar results to Agar Dilution. The rest of the thirty-two isolates were VISA according to E-strip, only one isolate was VISA as per Agar Dilution which again hints at the decreased accuracy of E-strips 
as compared to the Gold standard. Another study done by Holmes ${ }^{17}$ leads to similar conclusions.

While in ourstudy E-strip showed lower MIC as compared to Agar Dilution which further questions the precision and reliability of the method. Supporting our findings is another study 18 done in our region, in which $17 \%$ isolates out of 100 MRSA were reported as VISA using only Agar Dilution as detecting tool for VISA/VRSA. In Pakistan, a study done in Rawalpindi a few years back byKaleem et al ${ }^{19}$ was not able to detect any VISA or VRSA using onlythe E-strip method with no confirmation done using the gold standard method. This shows that E-strip might miss out VISA or VRSA, while Agar Dilution is more reliable when it comes to detecting VISA in MRSA isolates and thus can prevent under reporting and over reporting 5 . Another study concluded that before confirming results using commercial methods, they should be rechecked by reference methods to rectify the disparities in these detecting tools ${ }^{20}$.

VISA is on the rise regionally as well as globally. While the VISA percentage in a few countries recently has been reported upto $13 \%$ and $11 \%{ }^{21}$, VRSA is still rare ${ }^{22}$, But the pressure selection however, which has led to the emergence of VISA can create an alarming situation leading to complete resistance to Vancomycin (VRSA), which will not only be a global health hazard but also a fearsome threat to already challenging therapy of MRSA.

\section{RECOMMENDATION}

The evidence of MRSA strains showing VISA and VRSA have added peril to health care communities and thus preventive measures can play a pivotal role in limiting its spread.

Hand washing is the single most effective and simple way of its prevention especially in the hospitals. Similarly controlling the infections from spreading within a hospital or a community should be the first step, which can not only prevent the disease itself but also limit the use of anti-microbials used for the killing the bug.
Judicious use of anti-microbials is very important in this era of emerging resistance. Excessive use can lead to resistant strains with increased morbidity and mortality.

Last but not the least, use of right method for calculating MIC is a critical part of the equation. Agar Dilution should be used to evaluate the MIC of Vancomycin in MRSA isolates, so that not even a simple case of VISA or VRSA is missed or under reported. This will help to counter not only the emergence of mutated strains but also the treatment failures that might accompany the MIC creep.

\section{CONCLUSION}

E-strip has a high sensitivity and accuracy as compared to Agar Dilution in calculating the MIC of Vancomycin in MRSA isolates. However, its inability to identify VISA limits its usage as a method at par with the gold standard.

\section{CONFLICT OF INTEREST}

This study has no conflict of interest to declare by any author.

\section{REFERENCES}

1. Song JH, Hsueh PR, Chung DR, Ko KS, Kang CI, Peck KR, et al. Spread of methicillin-resistant Staphylococcus aureus between the community and the hospitals in Asian countries: an ANSORP study. J Antimicrob Chemother 2011; 66(5): 1061-69.

2. Kshetry AO, Pant ND, Bhandari R, Khatri S, Shrestha KL, Upadhaya SK, et al. Minimum inhibitory concentration of vancomycin to methicillin resistant Staphylococcus aureus isolated from different clinical samples at a tertiary care hospital in Nepal. Antimicrob Resist Infect Control 2016; 5(1): 27.

3. Finks JF, Wells E, Dyke TL, Husain N, Plizga L. Vancomy-cinresistant Staphylococcus aureus, Michigan, USA, 2007. Emerg Infect Dis 2009; 15(6): 943.

4. Kale SS, Patil A. The study of reduced susceptibility of methicillin resistant Staphylococcus aureus to various antibiotics with special reference to glycopeptides in a tertiary care hospital in central India. Int J Community Med Public Health 2019; 6(4): 1426.

5. Tandel K, Praharaj AK, Kumar S. Differences in vancomycin MIC among MRSA isolates by agar dilution and E test method. Indian J Med Microbiol 2012; 30(4): 453.

6. Dhand A, Sakoulas G. Reduced vancomycin susceptibility among clinical Staphylococcus aureus isolates ('the MIC Creep'): implications for therapy. F1000 Medicine Reports 2012; 4(1): 1-9.

7. Chen SY, Liao CH, Wang JL, Chiang WC, Lai MS et al. Methodspecific performance of vancomycin MIC susceptibility tests in predicting mortality of patients with methicillin-resistant Staphylococcus aureus bacteraemia. J Antimicrobial Chemotherapy 2014; 69(1): 211-18. 
8. Amatya R, Devkota P, Gautam A. Reduced susceptibility to Vancomycin in methicillin resistant Staphylococccusaureus: a time for action. Nepal Med Coll J 2014; 16(1): 42-44.

9. Kumari J, Shenoy MS, Chakrapani M, Vidyalakshmi K, Bhat KG. Comparison of $\mathrm{E}$ test and agar dilution for determining minimum inhibitory concentration of vancomycin to healthcareassociated methicillin-resistant Staphylococcus aureus. Asian J Pharm Clin Res 2016; 9(4): 189-91.

10. Phillips CJ, Wells NA, Martinello M, Smith S, Woodman RJ, Gordon DL. Optimizing the detection of methicillin-resistant Staphylococcus aureus with elevated vancomycin minimum inhibitory concentrations within the susceptible range. Infect Drug Resist 2016; 9(1): 87-92.

11. Kaleem F, Usman J, Sattar A, Amanat ST, Hassan A, Omair M, et al. Current status of vancomycin susceptibility against methicillin resistant Staphylococcus aureus (MRSA) strains: A study at two tertiary care hospitals of Pakistan. Afr. J Microbiol Res 2012; 6(33): 6243-46.

12. Mirza HC. Glycopeptide resistance in S. aureus. The rise of virulence and antibiotic resistance in staphylococcus aureus. Croatia: InTech. 2017: 43-59.

13. Hsu DI, Hidayat LK, Quist R, Hindler J, Karlsson A, Yusof A, et al. Comparison of method-specific vancomycin minimum inhibitory concentration values and their predictability for treatment outcome of meticillin-resistant Staphylococcus aureus (MRSA) infections. Int J Antimicrob Agents 2008; 32(5): 378-85.

14. Chaudhari CN, Tandel K, Grover N, Bhatt P, Sahni AK, Sen S, et al. In vitro vancomycin susceptibility amongst methicillin resistant Staphylococcus aureus. Med J Armed Forces India 2014; 70(3): 215-19.

15. Sakoulas G, Moellering RC. Methicillin-resistant Staphylococcus aureus (MRSA): increasing antibiotic resistance. Clin Infect Dis 2008; 46(Suppl 5): S360-67.

16. Wang JL, Wang JT, Sheng WH, Chen YC, Chang SC. Nosocomial methicillin-resistant Staphylococcus aureus (MRSA) bacteremia in Taiwan: mortality analyses and the impact of vancomycin, MIC $=2 \mathrm{mg} / \mathrm{L}$, by the broth microdilution method. BMC Infect Dis 2010; 10(1): 159-62.

17. Holmes NE, Turnidge JD, Munckhof WJ, Robinson JO, Korman $\mathrm{TM}$, O'Sullivan MV, et al. Vancomycin AUC/MIC ratio and 30day mortality in patients with Staphylococcus aureus bacteremia. Antimicrob Agents Chemother 2013; 57(4): 1654-63.

18. Reddy PS, John MS, Devi PV, Reddy BS. Detection of vancomycin susceptibility among clinical isolates of MRSA by using minimum inhibitory concentration method. Int J Res Med Sci 2015; 3(6): 1378-82.

19. Kaleem F, Usman J, Sattar A, Amanat ST, Hassan A, Omair M, et al. Current status of vancomycin susceptibility against methicillin resistant Staphylococcus aureus (MRSA) strains: A study at two tertiary care hospitals of Pakistan. Afr J Microbiol Res 2012; 6(33): 6243-46.

20. Kumari J, Shenoy SM, Hegde A, Vidyalakshmi K, Chakrapani M, Bhat GK. Vancomycin intermediate and vancomycin resistant staphylococcus aureus-mechanisms, clinical significance, and detection. Asian J Pharma Clin Res 2017; 10(6): 32-38.

21. Ghias W, Sharif M, Yazdani FA, Rabbani M. Isolation and identification of Methicillin and Vancomycin resistance Staphylococcus aureus from pus samples of injured skin patients in Lahore, Pakistan. Biomed Lett 2016; 2(2): 103-12.

22. McGuinness WA, Malachowa N, DeLeo FR. Focus: infectious diseases: vancomycin resistance in Staphylococcus aureus. Yale J Biol Med 2017; 90(2): 269-81. 